\title{
A ionized reflecting skin above the accretion disk of GX $349+2$
}

\author{
R. $\operatorname{Iaria}^{1}$, A. D’Aî́ ${ }^{1}$, T. Di Salvo ${ }^{1}$, N. R. Robba ${ }^{1}$, A. Riggio ${ }^{2}$, A. Papitto ${ }^{2}$, and L. Burderi ${ }^{2}$ \\ 1 Dipartimento di Scienze Fisiche ed Astronomiche, Università di Palermo, via Archirafi 36, 90123 Palermo, Italy \\ e-mail: iaria@isica.unipa.it \\ 2 Dipartimento di Fisica, Università degli Studi di Cagliari, SP Monserrato-Sestu, KM 0.7, 09042 Monserrato, Italy
}

Received 24 February 2009 / Accepted 13 June 2009

\section{ABSTRACT}

\begin{abstract}
Context. The broad emission features in the $\mathrm{Fe}-\mathrm{K} \alpha$ region of X-ray binary spectra represent an invaluable probe to constrain the geometry and the physics of these systems. Several Low Mass X-ray binary systems (LMXBs) containing a neutron star (NS) show broad emission features between 6 and $7 \mathrm{keV}$ and most of them are now interpreted as reflection features from the inner part of an accretion disk, in analogy to those observed in the spectra of X-ray binary systems containing a black hole candidate.

Aims. The NS LMXB GX 349+2 was observed by the XMM-Newton satellite which allows, thanks to its high effective area and good spectral resolution between 6 and $7 \mathrm{keV}$, a detailed spectroscopic study of the $\mathrm{Fe}-\mathrm{K} \alpha$ region.

Methods. We study the XMM data in the $0.7-10 \mathrm{keV}$ energy band. The continuum emission is modelled by a blackbody component plus a multicolored disk blackbody. A very intense emission line at $1 \mathrm{keV}$, three broad emission features at $2.63,3.32,3.9 \mathrm{keV}$ and a broader emission feature in the $\mathrm{Fe}-\mathrm{K} \alpha$ region are present in the residuals. The broad emission features above $2 \mathrm{keV}$ can be equivalently well fitted with Gaussian profiles or relativistic smeared lines (diskline in XSPEC). The Fe-K $\alpha$ feature is better fitted using a diskline component at $6.76 \mathrm{keV}$ or two diskline components at 6.7 and $6.97 \mathrm{keV}$, respectively.

Results. The emission features are interpreted as resonant transitions of S XVI, Ar XVIII, Ca XIX, and highly ionized iron. Modelling the line profiles with relativistic smeared lines, we find that the reflecting plasma is located at less than $40 \mathrm{~km}$ from the NS, a value compatible with the inner radius of the accretion disk inferred from the multicolored disk blackbody component $(24 \pm 7 \mathrm{~km})$. The inclination angle of GX $349+2$ is between $40^{\circ}$ and $47^{\circ}$, the emissivity index of the primary emission is between -2.4 and -2 , and the reflecting plasma extends up to $(2-8) \times 10^{8} \mathrm{~cm}$.

Conclusions. We compare our results with the twin source Sco X-1 and with the other NS LMXBs showing broad relativistic lines in their spectra. We conclude that the blackbody component in the spectrum is the primary emission that hits the inner accretion disk producing the emission lines broadened by relativistic and Doppler effects dominant around the neutron star.
\end{abstract}

Key words. line: identification - line: formation - stars: individual: GX 349+2 - X-rays: binaries - X-rays: general

\section{Introduction}

Low-mass X-ray binaries (LMXBs) consist of a low-mass star $\left(M<1 M_{\odot}\right)$ and a neutron star $(\mathrm{NS})$, which generally has a relatively weak magnetic field $\left(B<10^{10} \mathrm{G}\right)$. In these systems, the X-ray source is powered by accretion of mass overflowing the Roche lobe of the companion star and forming an accretion disk around the neutron star. LMXBs containing a neutron star (NS LMXBs) are generally divided into Z and Atoll sources, according to the path they describe in an X-ray color-color diagram (CD) or hardness-intensity diagram (Hasinger \& van der Klis 1989) assembled by using the source count rate and colors calculated over a typical (usually $2-20 \mathrm{keV}$ ) X-ray energy range. Atoll sources are usually characterized by relatively low luminosities (0.01-0.2 $\left.L_{\text {Edd }}\right)$ and often show transient behavior, while the $\mathrm{Z}$ sources in the Galaxy are among the most luminous LMXBs, persistently accreting close to the Eddington limit ( $\left.L_{\text {Edd }}\right)$ for a $1.4 M_{\odot}$ NS. The position of an individual source in the CD, which determines most of the observed spectral and temporal properties of the source, is thought to be an indicator of the instantaneous mass accretion rate (e.g. Hasinger et al. 1990; van der Klis 1995, for a review). It has been suggested that the mass accretion rate (but not necessarily the X-ray luminosity) of individual sources increases along the track from the top left to the bottom right, i.e. from the islands to the banana branch in atoll sources and from the horizontal branch (hereafter HB) to the normal branch (NB) and to the flaring branch $(\mathrm{FB})$ in $\mathrm{Z}$ sources.

GX 349+2, also known as Sco X-2, was called a peculiar case among the Z sources (Kuulkers \& van der Klis 1998). Similar to the case of Sco X-1, GX 349+2 shows a short and underdeveloped HB (if at all). The source variability in the frequency range below $100 \mathrm{~Hz}$ is closely correlated with the source position on the X-ray CD, as in other Z sources. Quasi periodic oscillations at $\mathrm{kHz}$ frequencies $(\mathrm{kHz} \mathrm{QPO})$ were detected in the NB of its Z-track (Zhang et al. 1998). However, GX 349+2, which sometimes shows broad noise components changing not only with the position in the Z, but also as a function of the position in the hardness-intensity diagram, differs somewhat from the other $\mathrm{Z}$ sources and shows similarities to the behavior seen in bright atoll sources, such as GX 13+1 and GX 3+1 (Kuulkers \& van der Klis 1998; O’Neill et al. 2001, 2002).

Di Salvo et al. (2001), using BeppoSAX data, showed that the source energy spectrum below $30 \mathrm{keV}$ could be well fit by a blackbody (with a temperature of $0.5-0.6 \mathrm{keV}$ ) and a Comptonized component (with seed-photon temperature of $1 \mathrm{keV}$ and electron temperature of $2.7 \mathrm{keV}$ ). Three discrete features were observed in the spectrum: an emission line at $1.2 \mathrm{keV}$, probably associated with L-shell Fe XXIV or Ly- $\alpha$ Ne X, an emission line at $6.7 \mathrm{keV}(\mathrm{Fe} \mathrm{XXV})$ and an absorption edge at $8.5 \mathrm{keV}$, both corresponding to emission from the $\mathrm{K}$-shell 
of highly-ionized iron (Fe XXV). Iaria et al. (2004), analysing a long BeppoSAX observation, found similar parameters of the the continuum components below $30 \mathrm{keV}$, detected the presence of emission lines associated with Fe XXIV and Fe XXV, and detected an emission line at $2.65 \mathrm{keV}$ associated with $\mathrm{S} X \mathrm{XVI}$. The long BeppoSAX observation allowed us to study changes of the parameters of the Fe XXV emission line along the position of the source in the $\mathrm{CD}$ from the NB/FB apex to the FB, inferring that the equivalent width of the line decreases from $77 \mathrm{eV}$ to $18 \mathrm{eV}$ going from the NB/FB apex to the FB. The width of the Fe XXV emission line, modelled by a Gaussian line, was between 250 and $300 \mathrm{eV}$.

Cackett et al. (2008), analysing two Suzaku observations of GX 349+2, modelled the Fe XXV emission line with a relativistic smeared line (diskline in XSPEC). The authors constrained the energy of the line to be between 6.4 and $6.97 \mathrm{keV}$, finding that the line energy was $6.97_{-0.02} \mathrm{keV}$, associated with Fe XXVI and not compatible with a Fe XXV iron line as previously obtained by Iaria et al. (2004) and Di Salvo et al. (2001). The emissivity index, $\beta$, was $-4.1 \pm 0.3$, the inner radius in gravitational radii, $R_{\mathrm{g}}$, was $8.0 \pm 0.4$, the inclination angle of the source was $23 \pm$ 1 degrees, the equivalent width of the line was $76 \pm 6 \mathrm{eV}$.

In this paper, we analyse an XMM-Newton observation of GX 349+2, finding that the prominent relativistic line is well constrained around $6.7 \mathrm{keV}$ and identified as the resonant transition of Fe XXV. In the following, we discuss our results and compare them with the recent literature.

\section{Observation}

The XMM-Newton Observatory (Jansen et al. 2001) includes three $1500 \mathrm{~cm}^{2} \mathrm{X}$-ray telescopes each with an European Photon Imaging Camera (EPIC, $0.1-15 \mathrm{keV}$ ) at the focus. Two of the EPIC imaging spectrometers use MOS CCDs (Turner et al. 2001) and one uses pn CCDs (Strüder et al. 2001). Reflection grating spectrometers (RGS, 0.35-2.5 keV, den Herder et al. 2001) are located behind two of the telescopes. The region of sky containing GX 349+2 was observed by XMM-Newton between 2008 March 19 16:42:41 UT to March 19 22:58:55 UT (OBSid 0506110101 ) for a duration of $22.5 \mathrm{ks}$. Since GX $349+2$ is one of the brightest X-ray sources, in order to minimize the effects of telemetry saturation and pile-up, the MOS1 and MOS2 instruments were switched off and the EPIC-pn camera was operated in Timing mode with medium filter during the observation. In this mode, only one central CCD is read out with a time resolution of $0.03 \mathrm{~ms}$. This provides a one dimensional (4.4 wide) image of the source with the second spatial dimension being replaced by timing information. The faster CCD readout results in a much higher count rate capability of $1500 \mathrm{cts} / \mathrm{s}$ before charge pile-up becomes a serious problem for point-like sources. The EPIC-pn telemetry limit is approximatively $450 \mathrm{c} / \mathrm{s}$ for the timing mode ${ }^{1}$. If the rate is higher, then the counting mode is triggered and part of the science data are lost. This is the case for the observation presented in this work; saturation occurs for $55 \%$ of the observing time, giving an exposure time of $22.5 \mathrm{ks}$ for RGS1 and RGS2, respectively, and 10 ks for EPIC-pn.

We plot the Epic-pn lightcurve in the 1-10 keV energy range in Fig. 1 which clearly shows that the count rate is largely variable. To understand whether the variability of the source intensity is energy dependent, we extract the lightcurves in the $1-4 \mathrm{keV}$ and $4-10 \mathrm{keV}$ energy range, respectively, and plot the

\footnotetext{
${ }^{1}$ See Table 3 in the XMM-Newton Users Handbook published on 15 July 2008 .
}



Fig. 1. Epic-pn lightcurve of GX $349+2$ in the energy band 1-10 keV. The bin time is $30 \mathrm{~s}$.

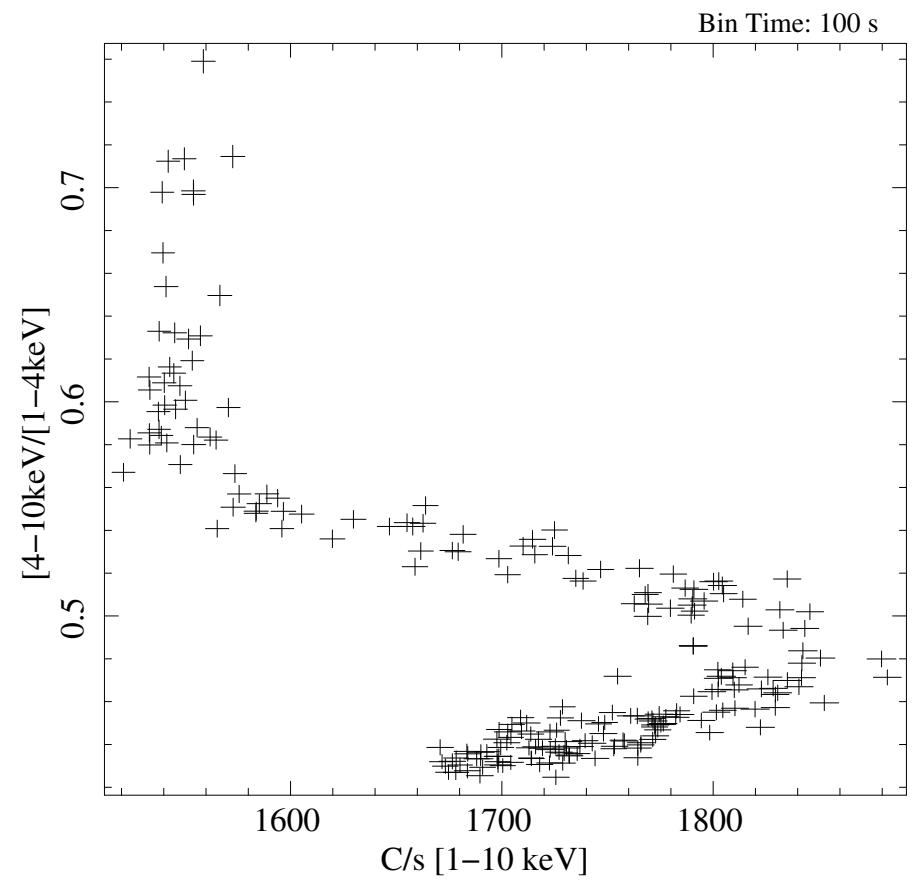

Fig. 2. Color-intensity diagram of GX $349+2$. The $[4-10 \mathrm{keV}] /[1-$ $4 \mathrm{keV}$ ] hardness ratio increases for a count rate lower than $1580 \mathrm{c} / \mathrm{s}$. The bin time is $100 \mathrm{~s}$.

hardness ratio [4-10 $\mathrm{keV}] /[1-4 \mathrm{keV}]$ versus the count rate in the $1-10 \mathrm{keV}$ energy range in Fig. 2. We find that the spectrum becomes harder for a count rate below $1580 \mathrm{c} / \mathrm{s}$ corresponding to the time interval between $10^{4}$ and $1.5 \times 10^{4} \mathrm{~s}$ from the start of the observation. Plotting the $1-4 \mathrm{keV}, 4-10 \mathrm{keV}$ lightcurve and the [4-10 keV]/[1-4 keV] hardness ratio versus time (see Fig. 3), it seems that there is no correlation between the $1-4 \mathrm{keV}$ and 4-10 keV count rate. The $1-4 \mathrm{keV}$ count rate is quite constant at $1200 \mathrm{c} / \mathrm{s}$ between 0 and $9 \times 10^{3} \mathrm{~s}$ and between $1.5 \times 10^{4}$ and 


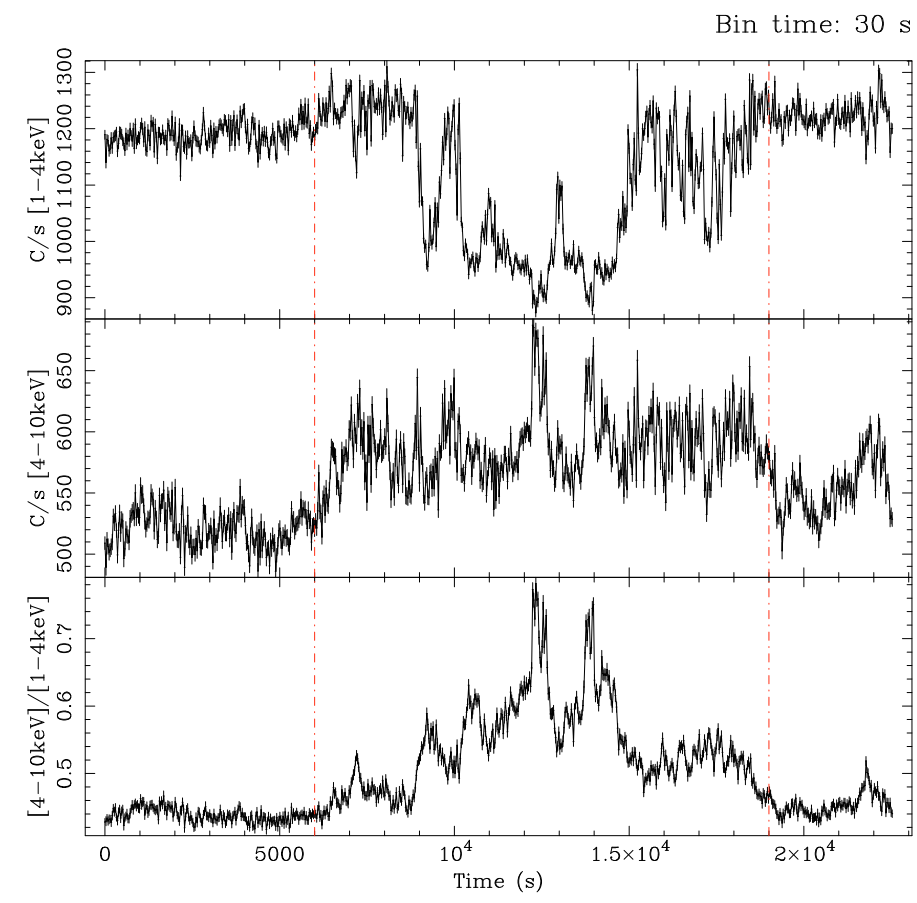

Fig. 3. Upper panel: 1-4 keV Epic-pn lightcurve. Middle panel: 4-10 keV Epic-pn lightcurve. Bottom panel: [4-10 keV]/[1-4 keV] hardness ratio. The bin time is $30 \mathrm{~s}$ for each lightcurve. The red dotdashed vertical lines indicate the three selected time intervals.

Table 1. Time intervals selected in the EPIC-pn lightcurve.

\begin{tabular}{lccc}
\hline \hline & $\begin{array}{c}\text { Time interval } \\
\text { s - TSTART[s] }\end{array}$ & $\begin{array}{c}\text { RGS1 } \\
\text { ks }\end{array}$ & $\begin{array}{c}\text { EPIC-pn } \\
\text { ks }\end{array}$ \\
\hline INT. 1 & $0-6000$ & 6.0 & 2.6 \\
INT. 2 & $6000-19000$ & 13.0 & 5.8 \\
INT. 3 & $>19000$ & 3.5 & 1.6 \\
\hline
\end{tabular}

Note. The observation was divided into three time intervals as shown in Fig. 3. In the first column we report the temporal boundaries of each interval with respect to the start time of the observation. In the second and third column we report the exposure times, in kiloseconds, for RGS1, RGS2, and EPIC-pn during the three time intervals.

$2.2 \times 10^{4} \mathrm{~s}$ from the start, and it decreases between $9 \times 10^{3}$ and $1.5 \times 10^{4} \mathrm{~s}$, showing some flaring activity. The $4-10 \mathrm{keV}$ count rate is quite constant at $530-550 \mathrm{c} / \mathrm{s}$ between 0 and $6 \times 10^{3} \mathrm{~s}$ and between $1.9 \times 10^{4}$ and $2.25 \times 10^{4} \mathrm{~s}$, while it increases to $600 \mathrm{c} / \mathrm{s}$ between $6 \times 10^{3}$ and $1.9 \times 10^{4} \mathrm{~s}$.

Since the count rate of the source is variable, we divide the lightcurve into three time intervals as indicated in Fig. 3 by the red dot-dashed lines. We base our choice on the 4-10 keV count rate that is $550 \mathrm{c} / \mathrm{s}$ in the first and third time interval and around $600 \mathrm{c} / \mathrm{s}$ in the second time interval. Our choice allows us to highlight possible changes in the $\mathrm{Fe}-\mathrm{K} \alpha$ region of the spectrum. In Table 1 we report the exposure times of the selected time intervals for RGS1, RGS2 and EPIC-pn instruments.

\subsection{Spectral analysis of the averaged spectrum}

We extract the X-ray data products of the RGS and EPIC-pn cameras using the Science Analysis Software (SAS) version 8.0.0, extracting only single and double events (patterns 0 to 4 ) from EPIC-pn data. Initially we extracted source EPIC-pn events from a 69.7" wide column (RAWX between 29 and 45) centered
Table 2. Percentage of photon pile-up at different energies.

\begin{tabular}{|c|c|c|}
\hline $\begin{array}{l}\text { Energy } \\
(\mathrm{keV})\end{array}$ & $\begin{array}{c}\text { Pile-up (\%) } \\
\text { RAWX [29:45] }\end{array}$ & $\begin{array}{c}\text { Pile-up (\%) } \\
\text { RAWX [29:36] and RAWX[39:45] }\end{array}$ \\
\hline 5 & $1.3 \pm 0.1$ & $0.2 \pm 0.2$ \\
\hline 6 & $2.0 \pm 0.1$ & $0.6 \pm 0.2$ \\
\hline 7 & $3.2 \pm 0.2$ & $1.6 \pm 0.2$ \\
\hline 8 & $5.1 \pm 0.3$ & $1.9 \pm 0.4$ \\
\hline 9 & $7.4 \pm 0.4$ & $4.8 \pm 0.5$ \\
\hline 10 & $9.8 \pm 0.6$ & $6.5 \pm 0.8$ \\
\hline
\end{tabular}

Note. The associated errors are reported at $1 \sigma$. In the first column we report the energies, in the second and third column the estimated percentage of photon pile-up, extracting the events from the whole source region and excluding the brightest columns at RAWX $=37$ and RAWX $=38$, respectively.

on the source position (RAWX $=37$ ). Background events were obtained from a box of the same width with RAWX between 2 and 18.

The $0.5-12 \mathrm{keV}$ Epic-pn count rate extracted from the source region is almost $1800 \mathrm{c} / \mathrm{s}$; since the maximum Epic-pn count rate should be $800 \mathrm{c} / \mathrm{s}$ to avoid a deteriorated response due to photon pile-up ${ }^{2}$, we expect the presence of pile-up. We checked the pileup fraction at different energies using the epatplot tool in SAS, finding a photon pile-up percentage of $1.3 \%, 2 \%, 3.2 \%, 5.1 \%$, $7.4 \%$, and $9.8 \%$ at $5,6,7,8,9$, and $10 \mathrm{keV}$, respectively. The values with the associated errors are reported in Table 2. Since our aim is the study of the $\mathrm{Fe}-\mathrm{K} \alpha$ region of the spectrum, we investigated how to minimize the photon pile-up effects in the energy range 5-8 keV. Initially we extracted the source EPICpn spectrum, excluding the brightest column ( RAWX $=37$ ) in the CCD. The photon pile-up fraction did not change significantly; we obtained a photon pile-up percentage of $1.1 \%$, $1.7 \%, 3.1 \%, 4.7 \%, 6.7 \%$, and $9.2 \%$ at $5,6,7,8,9$, and $10 \mathrm{keV}$. Finally, excluding the two brightest columns at RAWX $=37$ and RAWX $=38$, we found a photon pile-up fraction of $0.2 \%, 0.6 \%$, $1.6 \%, 1.9 \%, 4.8 \%$, and $6.5 \%$ at $5,6,7,8,9$, and $10 \mathrm{keV}$. In this case, the $0.5-12 \mathrm{keV}$ Epic-pn count rate is $920 \mathrm{c} / \mathrm{s}$. The photon pile-up percentage values with the associated errors are reported in Table 2 and the plot of the fraction patterns of single and double events are shown in Fig. 4. After this investigation, we chose to extract the Epic-pn spectrum excluding the two brightest columns at RAWX $=37$ and RAWX $=38$ from the source region to avoid any doubt on the validity of our analysis.

Since the high available statistics, the EPIC-pn spectrum shows evident calibration issues between 2 and $2.5 \mathrm{keV}$ due to the $\mathrm{Au}$ edge near $2.3 \mathrm{keV}$, we excluded the EPIC-pn data between 2.1 and $2.5 \mathrm{keV}$ from our analysis. The EPIC-pn spectrum has been rebinned so as not to oversample the energy resolution by more than a factor of 4 and to have 20 counts per energy channel. The adopted Epic-pn energy range is $0.7-2.1 \mathrm{keV}$ and $2.5-10 \mathrm{keV}$. The spectrum is fitted using XSPEC version 12.3.1

The extracted RGS1 spectrum does not show instrumental issues and we select data in the $0.6-2 \mathrm{keV}$ energy band for our analysis. The extracted RGS2 spectrum shows calibration problems for CCD 9 and we will not use data from this instrument for our analysis. Since we are interested in the study of the Fe$\mathrm{K} \alpha$ region, in the following we concentrate our spectral analysis on the Epic-pn data, after having checked that the RGS1 spectrum is consistent with the Epic-pn data and does not show more features.

\footnotetext{
${ }^{2}$ See Table 3 in the XMM-Newton Users Handbook published on 15 July 2008
} 


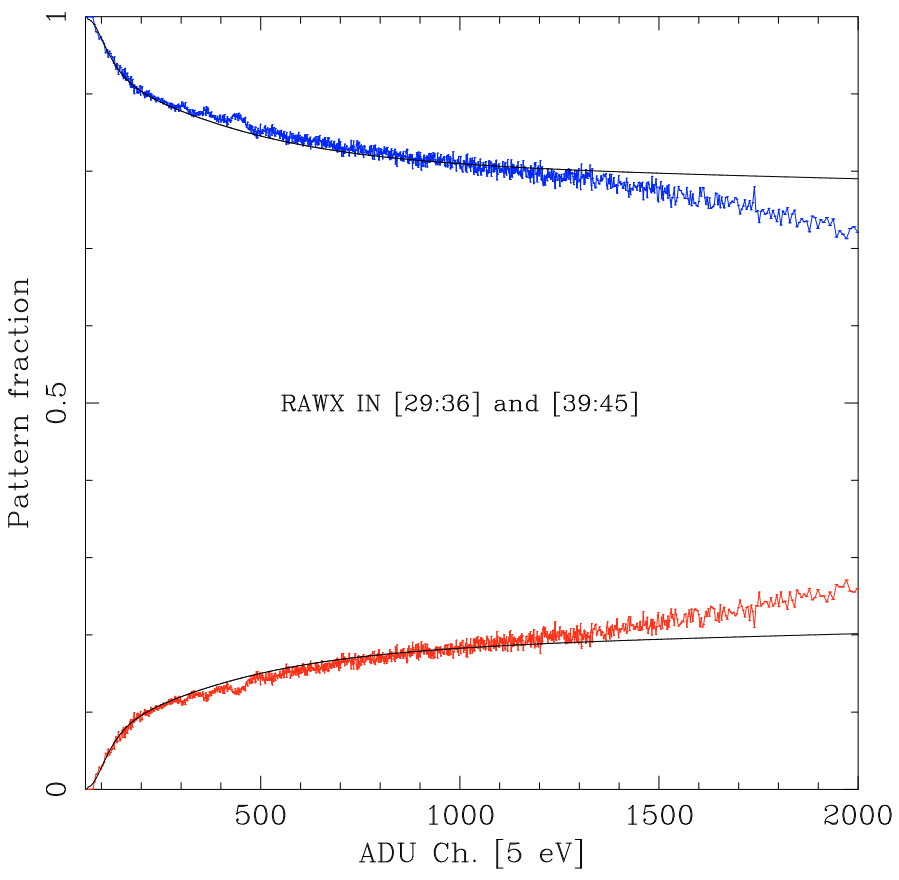

Fig. 4. Patterns of single (blue color) and double (red color) events extracted from the source region excluding RAWX 37 and 38. The patterns are plotted versus the 60-2000 ADU channel range, corresponding to $0.3-10 \mathrm{keV}$.

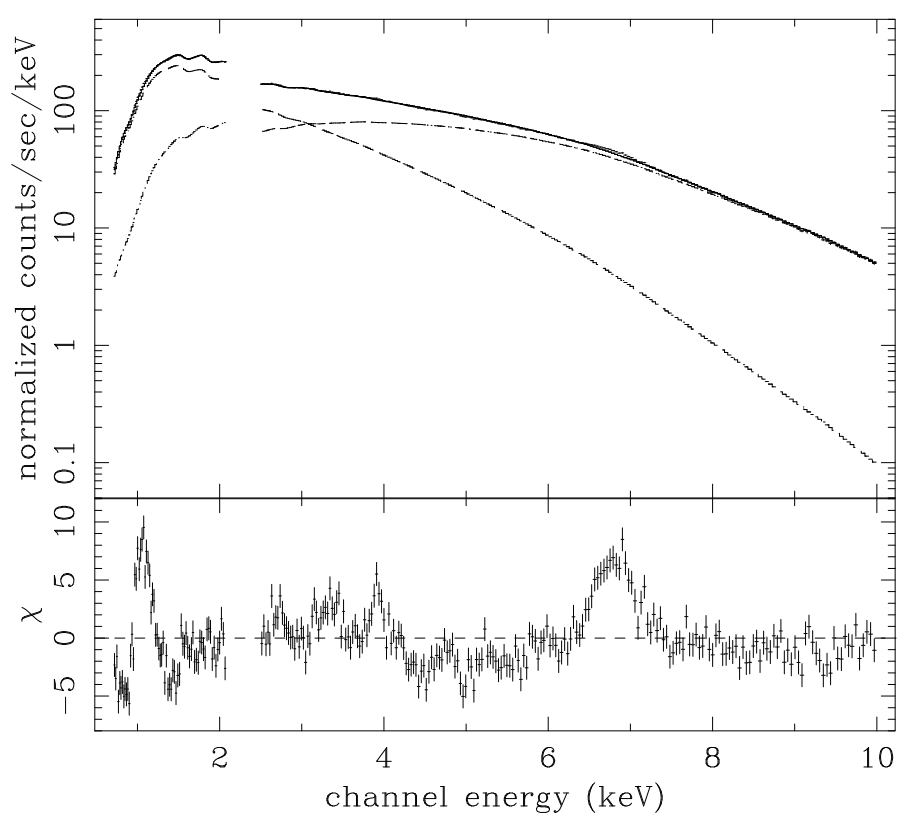

Fig. 5. Residuals (in units of $\sigma$ ) in the $0.7-10 \mathrm{keV}$ energy range. The adopted continuum is composed of blackbody plus a multicolored disk blackbody, both absorbed by neutral matter. In the residuals, clear emission features are evident at 1, 2.7, 3.35, 3.9 and in the $\mathrm{Fe}-\mathrm{K} \alpha$ region of the spectrum.

The continuum emission was fitted adopting the model by Cackett et al. (2008) for the Suzaku spectrum of GX 349+2: i.e. a blackbody component plus a multicolored disk blackbody (DISKBB in XSPEC) both absorbed by neutral neutral matter (WABS in XSPEC); because of the narrower energy range of our Epic-pn data $(0.7-10 \mathrm{keV})$ with respect to the Suzaku data we did not add to the model a power-law component necessary
Table 3. Averaged continuum and identified broad Gaussian lines below $6 \mathrm{keV}$.

\begin{tabular}{|c|c|c|}
\hline Parameters & $\begin{array}{l}\text { Single Fe-K } \alpha \\
\text { Em. Line }\end{array}$ & $\begin{array}{c}\text { Fe XXV-Fe XXVI } \\
\text { Blending }\end{array}$ \\
\hline$N_{\mathrm{H}}\left(10^{22} \mathrm{~cm}^{-2}\right)$ & $0.735_{-0.003}^{+0.006}$ & $0.736_{-0.004}^{+0.005}$ \\
\hline $\begin{array}{l}k T_{\mathrm{BB}}(\mathrm{keV}) \\
N_{\mathrm{BB}}\left(10^{-1}\right)\end{array}$ & $\begin{array}{l}1.792_{-0.019}^{+0.006} \\
1.052_{-0.009}^{+0.017}\end{array}$ & $\begin{array}{l}1.787_{-0.014}^{+0.015} \\
1.057_{-0.014}^{+0.012}\end{array}$ \\
\hline $\begin{array}{l}k T_{\text {DISKBв }}(\mathrm{keV}) \\
N_{\text {DISKBB }}\end{array}$ & $\begin{array}{c}1.05_{-0.03}^{+0.02} \\
210_{-30}^{+10}\end{array}$ & $\begin{array}{c}1.04 \pm 0.03 \\
220 \pm 20\end{array}$ \\
\hline $\begin{array}{l}E_{1 \mathrm{keV}}(\mathrm{keV}) \\
\sigma_{1 \mathrm{keV}}(\mathrm{eV}) \\
I_{1 \mathrm{keV}}\left(10^{-3} \mathrm{ph} \mathrm{cm}^{-2} \mathrm{~s}^{-1}\right) \\
\text { Equiv. width }(\mathrm{eV}) \\
\text { significance }(\sigma)\end{array}$ & $\begin{array}{c}1.051_{-0.006}^{+0.006} \\
90_{-8}^{+11} \\
25 \pm 3 \\
22_{-3}^{+4} \\
14\end{array}$ & $\begin{array}{c}1.051_{-0.006}^{+0.006} \\
85_{-6}^{+12} \\
24_{-2}^{+4} \\
21 \pm 2 \\
18\end{array}$ \\
\hline $\begin{array}{l}E_{\mathrm{S} X V I}(\mathrm{keV}) \\
\sigma_{\mathrm{S} X \mathrm{XI}}(\mathrm{eV}) \\
I_{\mathrm{S} \text { XVI }}\left(10^{-3} \mathrm{ph} \mathrm{cm}^{-2} \mathrm{~s}^{-1}\right) \\
\text { Equiv. width }(\mathrm{eV}) \\
\text { significance }(\sigma)\end{array}$ & $\begin{array}{c}2.62 \text { (fixed) } \\
140 \text { (fixed) } \\
2.3_{-0.5}^{+0.9} \\
6 \pm 2 \\
7\end{array}$ & $\begin{array}{l}2.62 \text { (fixed) } \\
140 \text { (fixed) } \\
2.5_{-0.6}^{+0.7} \\
6 \pm 2 \\
7\end{array}$ \\
\hline $\begin{array}{l}E_{\mathrm{Ar} \text { XVIII }}(\mathrm{keV}) \\
\sigma_{\mathrm{Ar} \text { XVIII }}(\mathrm{eV}) \\
I_{\mathrm{Ar} \text { XVIII }}\left(10^{-3} \mathrm{ph} \mathrm{cm}^{-2} \mathrm{~s}^{-1}\right) \\
\text { Equiv. width }(\mathrm{eV}) \\
\text { significance }(\sigma)\end{array}$ & $\begin{array}{c}3.33_{-0.04}^{+0.03} \\
190_{-40}^{+70} \\
3.7_{-0.4}^{+1.7} \\
12 \pm 5 \\
15\end{array}$ & $\begin{array}{c}3.32_{-0.04}^{+0.03} \\
200_{-50}^{+60} \\
4.0_{-1.1}^{+1.4} \\
14_{-5}^{+4} \\
6\end{array}$ \\
\hline $\begin{array}{l}E_{\mathrm{CaXIX}}(\mathrm{keV}) \\
\sigma_{\mathrm{CaXIX}}(\mathrm{eV}) \\
I_{\mathrm{CaXIX}}\left(10^{-3} \mathrm{ph} \mathrm{cm}^{-2} \mathrm{~s}^{-1}\right) \\
\text { Equiv. width }(\mathrm{eV}) \\
\text { significance }(\sigma)\end{array}$ & $\begin{array}{c}3.93_{-0.02}^{+0.03} \\
100_{-30}^{+50} \\
2.2_{-0.4}^{+0.7} \\
9 \pm 3 \\
9\end{array}$ & $\begin{array}{c}3.93 \pm 0.02 \\
110_{-20}^{+50} \\
2.3_{-0.4}^{+0.6} \\
9_{-2}^{+3} \\
9\end{array}$ \\
\hline$\chi_{\text {red }}^{2}$ (d.o.f. $)$ & $1.51(269)$ & $1.51(268)$ \\
\hline
\end{tabular}

Note. Best-fit values of the parameters obtained for the averaged spectrum fitting the emission features with Gaussian profiles. Uncertainties are at the $90 \%$ confidence level for a single parameter. The parameters are defined as in XSPEC. The continuum emission is fitted with a blackbody plus a multicolored disk blackbody, diskbb. The four broad emission features are modelled adopting Gaussian components. All components are subject to interstellar absorption.

to fit the Suzaku data above $10 \mathrm{keV}$. We obtained a reduced $\chi_{\text {red }}^{2}=7.73$ with 282 degree of freedom (d.o.f.). The residuals, plotted in Fig. 5, suggest the presence of broad emission lines at $1,2.7,3.35,3.9 \mathrm{keV}$ and, finally a broader emission line between 6 and $7 \mathrm{keV}$. Initially, we added five Gaussian components to fit these emission features. Since the line around $2.7 \mathrm{keV}$ is near the hole in the Epic-pn data, we fixed the energy of the line at $2.62 \mathrm{keV}$, imposing that it is associated with the Ly$\alpha$ S XVI transition; furthermore we fixed its width at $140 \mathrm{eV}$. We obtain a $\chi_{\text {red }}^{2}$ (d.o.f.) $=1.51(269)$ and a $\chi^{2}$ improvement of $\Delta \chi^{2}=1775$. The significance in units of $\sigma$ of the lines at $1,2.62,3.32,3.9$, and $6.7 \mathrm{keV}$ is $14,7,15,9$, and 13 , respectively. The best-fit values are reported in Tables 3 (Col. 2) and 4 (Col. 2). We identify the broad emission lines centered at $1.051_{-0.015}^{+0.006} \mathrm{keV}, 3.33_{-0.04}^{+0.03} \mathrm{keV}$, and $3.93_{-0.02}^{+0.03} \mathrm{keV}$ as Fe XXII transition, Ly- $\alpha$ Ar XVIII transition, and resonance transition of CaXIX, respectively. The centroid of the broader line between 6 and $7 \mathrm{keV}$ is not identifiable because we find a line centered 


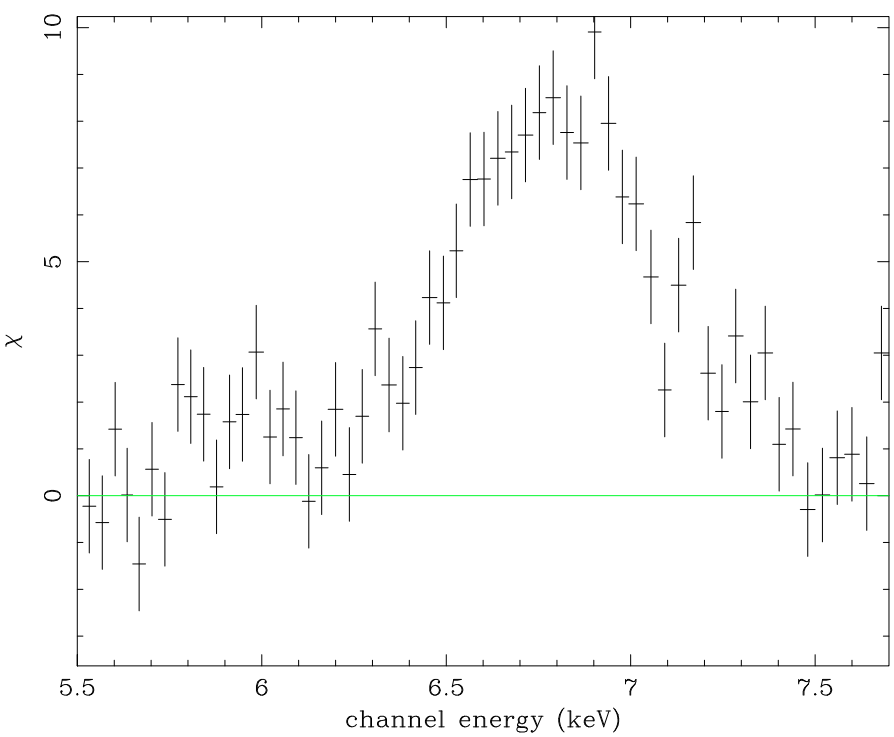

Fig. 6. Residuals in the 5.5-7.7 keV energy range. These can be fitted adopting a Gaussian profile or, equivalently, a relativistic smeared line (diskline in XSPEC).

Table 4. Broad Gaussian lines between 6 and $7 \mathrm{keV}$.

\begin{tabular}{|c|c|c|}
\hline Parameters & $\begin{array}{l}\text { Single Fe-K } \alpha \\
\text { Em. Line }\end{array}$ & $\begin{array}{c}\text { Fe XXV-Fe XXVI } \\
\text { Blending } \\
\end{array}$ \\
\hline$E_{\text {Broad }}(\mathrm{keV})$ & $6.80 \pm 0.02$ & - \\
\hline$\sigma_{\text {Broad }}(\mathrm{eV})$ & $280_{-40}^{+30}$ & - \\
\hline$I_{\text {Broad }}\left(10^{-3} \mathrm{ph} \mathrm{cm}^{-2} \mathrm{~s}^{-1}\right)$ & $4.7_{-0.4}^{+0.4}$ & - \\
\hline Equiv. width (eV) & $49_{-7}^{+6.0}$ & - \\
\hline significance $(\sigma)$ & 13 & - \\
\hline$E_{\mathrm{Fe} \mathrm{XXV}}(\mathrm{keV})$ & - & 6.70 (fixed) \\
\hline$\sigma_{\mathrm{Fe} \mathrm{XXV}}(\mathrm{eV})$ & - & $220_{-40}^{+20}$ \\
\hline$I_{\mathrm{Fe} \mathrm{XXV}}\left(10^{-3} \mathrm{ph} \mathrm{cm}^{-2} \mathrm{~s}^{-1}\right)$ & - & $2.6_{-0.6}^{+0.7}$ \\
\hline Equiv. width (eV) & - & $25 \pm 9$ \\
\hline significance $(\sigma)$ & - & 7 \\
\hline$E_{\mathrm{Fe} \mathrm{XXVI}}(\mathrm{keV})$ & - & 6.97 (fixed) \\
\hline$\sigma_{\mathrm{Fe} \text { XXVI }}(\mathrm{eV})$ & - & $280_{-70}^{+130}$ \\
\hline$I_{\mathrm{Fe} \mathrm{XXVI}}\left(10^{-3} \mathrm{ph} \mathrm{cm}^{-2} \mathrm{~s}^{-1}\right)$ & - & $2.0_{-0.6}^{+1.0}$ \\
\hline Equiv. width (eV) & - & $21 \pm 2$ \\
\hline significance $(\sigma)$ & - & 5 \\
\hline
\end{tabular}

Note. See Table 3.

at $6.80 \pm 0.02 \mathrm{keV}$. We also note that, while the FWHMs of the broad lines below $6 \mathrm{keV}$ are between 0.2 and $0.4 \mathrm{keV}$, the broader line in the $\mathrm{Fe}-\mathrm{K} \alpha$ region has a $F W H M$ of $0.6-0.7 \mathrm{keV}$ (the line profile is visible in the residuals shown in Fig. 6).

We have also investigated the possibility that the $\mathrm{Fe}-\mathrm{K} \alpha$ feature is a blending of lines. We fitted the broad $\mathrm{Fe}-\mathrm{K} \alpha$ emission feature as a blending of Fe XXV (6.7 keV) and Fe XXVI $(6.97 \mathrm{keV})$ emission lines adopting two Gaussian profiles and fixing their energies at the expected rest-frame values; the values of the fit are reported in Tables 3 (Col. 3) and 4 (Col. 3). Even in this case the Fe XXV and Fe XXVI emission lines appear to be broad, having widths of $200 \mathrm{eV}(F W H M=0.5 \mathrm{keV})$.

Under the hypothesis that the broadening of the lines is produced by the relativistic motion of the emitting plasma in the accretion disk close to the neutron star, we fit the line profiles at 2.62, 3.32, 3.90, and $6.80 \mathrm{keV}$ with relativistic disk line
Table 5. Best-fit values of the parameters obtained fitting the emission features with relativistic smeared lines (diskline in XSPEC). The Fe-K $\alpha$ feature is modelled with one diskline component.

\begin{tabular}{|c|c|c|c|}
\hline Aver. spectrum & Spectrum 1+3 & Spectrum 2 & \\
\hline$N_{\mathrm{H}}\left(10^{22} \mathrm{~cm}^{-2}\right)$ & $0.735 \pm 0.004$ & 0.735 (fixed) & 0.735 (fixed) \\
\hline$k T_{\mathrm{BB}}(\mathrm{keV})$ & $1.797_{-0.007}^{+0.016}$ & $1.780_{-0.009}^{+0.023}$ & $1.818_{-0.010}^{+0.013}$ \\
\hline$N_{\mathrm{BB}}\left(10^{-1}\right)$ & $1.048_{-0.013}^{+0.004}$ & $0.924_{-0.017}^{+0.0113}$ & $1.126_{-0.009}^{+0.007}$ \\
\hline$k T_{\text {DIS Квв }}(\mathrm{keV})$ & $1.049_{-0.014}^{+0.028}$ & $1.08_{-0.02}^{+0.03}$ & $1.05 \pm 0.02$ \\
\hline$N_{\text {DIS KBB }}$ & $207_{-20}^{+9}$ & $213_{-18}^{+14}$ & $190_{-12}^{+5}$ \\
\hline$E_{1 \mathrm{keV}}(\mathrm{keV})$ & $1.052_{-0.009}^{+0.006}$ & $1.030_{-0.016}^{+0.009}$ & $1.062_{-0.009}^{+0.004}$ \\
\hline$\sigma_{1 \mathrm{keV}}(\mathrm{eV})$ & $85_{-7}^{+13}$ & $120_{-8}^{+20}$ & $66_{-7}^{+10}$ \\
\hline$I_{1 \mathrm{keV}}$ & $24_{-2}^{+4}$ & $42_{-4}^{+8}$ & $17.0_{-1.4}^{+2.1}$ \\
\hline Equiv. width (eV) & $22 \pm 2$ & $32 \pm 2$ & $17 \pm 2^{-4}$ \\
\hline$\beta$ emiss. index & $-2.1 \pm 0.2$ & $-2.10_{-0.23}^{+0.12}$ & $-2.10_{-0.16}^{+0.11}$ \\
\hline$R_{\text {in }}\left(R_{\mathrm{g}}\right)$ & $6.2_{-0.2}^{+19.1}$ & $6.6_{-0.6}^{+12.2^{-}}$ & $10_{-4}^{+14}$ \\
\hline$R_{\text {out }}\left(R_{\mathrm{g}}\right)$ & $1980_{-710}^{+2900}$ & 1980 (fixed) & 1980 (fixed) \\
\hline$\theta$ (degrees) & $41.4_{-2.1}^{+1.0}$ & 41.4 (fixed) & 41.4 (fixed) \\
\hline$E_{\mathrm{S} X \mathrm{XI}}(\mathrm{keV})$ & 2.62 (fixed) & 2.62 (fixed) & 2.62 (fixed) \\
\hline$I_{\mathrm{S} X V I}$ & $2.2_{-0.9}^{+0.5}$ & $3.4_{-1.0}^{+0.9}$ & $0.9 \pm 0.6$ \\
\hline Equiv. width (eV) & $5 \pm 3$ & $8_{-3}^{+4}$ & $2_{-2}^{+3}$ \\
\hline$E_{\mathrm{Ar} \text { XVIII }}(\mathrm{keV})$ & $3.29 \pm 0.03$ & $3.32 \pm 0.02$ & $3.28_{-0.04}^{+0.02}$ \\
\hline$I_{\mathrm{Ar} X \mathrm{XVII}}$ & $3.5_{-0.6}^{+0.5}$ & $3.8_{-1.1}^{+0.9}$ & $2.7_{-0.8}^{+0.64}$ \\
\hline Equiv. width (eV) & $11 \pm 4$ & $12 \pm 4$ & $9 \pm 4$ \\
\hline$E_{\mathrm{Ca} \text { XIX }}(\mathrm{keV})$ & $3.89 \pm 0.03$ & $3.89_{-0.04}^{+0.03}$ & $3.90 \pm 0.03$ \\
\hline$I_{\mathrm{Ca} \mathrm{XIX}}$ & $3.6_{-0.7}^{+0.5}$ & $3.5 \pm 0.9$ & $3.2 \pm 0.7$ \\
\hline Equiv. width (eV) & $15 \pm 4$ & $15 \pm 5$ & $13 \pm 5$ \\
\hline$E_{\mathrm{Fe} X X V}(\mathrm{keV})$ & $6.76 \pm 0.02$ & $6.74 \pm 0.04$ & $6.78 \pm 0.03$ \\
\hline$I_{\mathrm{Fe} \mathrm{XXV}}$ & $5.7_{-0.6}^{+0.5}$ & $5.5 \pm 0.8$ & $5.8_{-0.8}^{+0.7}$ \\
\hline Equiv. width (eV) & $61 \pm 9$ & $64 \pm 11$ & $59 \pm 11$ \\
\hline$\chi_{\text {red }}^{2}$ (d.o.f.) & $1.47(268)$ & $1.24(271)$ & $1.30(271)$ \\
\hline
\end{tabular}

Note. Uncertainties are at the $90 \%$ confidence level for a single parameter. The parameters are defined as in XSPEC. The continuum emission is fitted with a blackbody plus a multicolored disk blackbody, diskbb. The four broad emission features are modelled adopting diskline components. All components are subject to interstellar absorption. The intensity of the lines are in units of $10^{-3} \mathrm{ph} \mathrm{cm}^{-2} \mathrm{~s}^{-1}$.

components (DISKLINE in XSPEC). Assuming that the emission features are produced in the same region, we impose that the inner radius, the outer radius, the emissivity index, and the inclination angle of the system are the same for all these components. Adding these components to the continuum emission we find a slightly better fit, $\chi_{\text {red }}^{2}$ (d.o.f.) $=1.47(268)$.

We find that the emissivity index of the accretion disk is -2.1 , the inner radius is less than 25 gravitational radii $\left(G M / c^{2}\right.$, hereafter $R_{\mathrm{g}}$ ), corresponding to $52 \mathrm{~km}$ for a NS mass of $1.4 M_{\odot}$, and the inclination angle of the system is $41^{\circ}$. The best-fit values of the parameters are reported in Table 5 (Col. 2); we plot data and residuals in the $0.7-10 \mathrm{keV}$ energy band and the corresponding unfolded spectrum in the $\mathrm{Fe}-\mathrm{K} \alpha$ region in Figs. 7 and 8 , respectively.

The Fe-K $\alpha$ line has an energy of $6.76 \pm 0.02 \mathrm{keV}$ and it is not compatible (at a $90 \%$ confidence level) with the rest-frame value of the Fe XXV transition $(6.7 \mathrm{keV})$. We therefore tried to fit the $\mathrm{Fe}-\mathrm{K} \alpha$ broad feature adopting two diskline components associated with Fe XXV and Fe XXVI transitions. We fixed the 


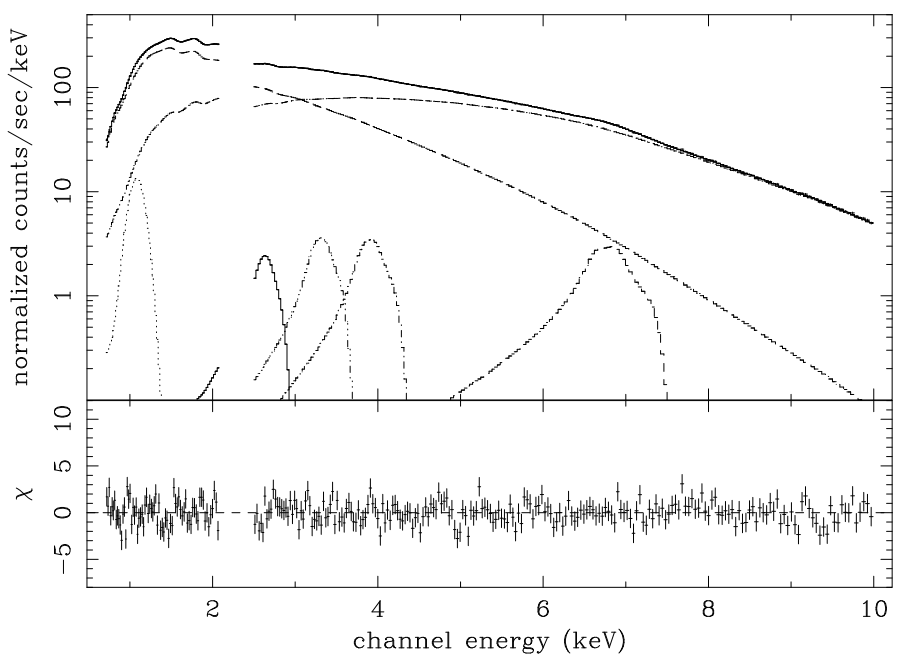

Fig. 7. Data, model, and residuals (in units of $\sigma$ ) in the $0.7-10 \mathrm{keV}$ energy range after adding the disk line components discussed in the text.

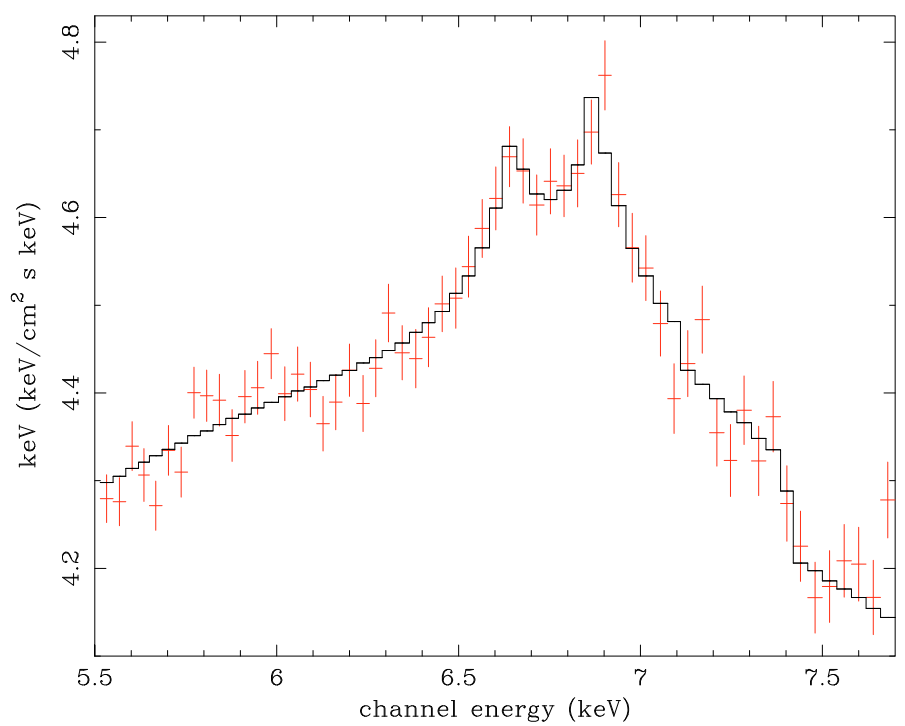

Fig. 8. Unfolded spectrum in the energy range 5.5-7.7 keV; the Fe XXV relativistic line profile is shown.

energy values at 6.7 and $6.97 \mathrm{keV}$, respectively. The best-fit parameters are reported in Table 6 (Col. 2); in this case we obtain $\chi_{\text {red }}^{2}$ (d.o.f.) $=1.48(268)$ and similar parameters of the emissivity index and inclination angle of the source. We find an emissivity index of $-2.2 \pm 0.2$, an inner radius less than $26 R_{\mathrm{g}}$, corresponding to $52 \mathrm{~km}$ for a NS mass of $1.4 M_{\odot}$, and an inclination angle of the system of $43_{-3}^{+4} \mathrm{deg}$.

The extrapolated absorbed and unabsorbed flux in the $0.1-100 \mathrm{keV}$ energy range was $1.2 \times 10^{-8}$ and $1.4 \times$ $10^{-8} \mathrm{erg} \mathrm{cm}^{-2} \mathrm{~s}^{-1}$, respectively. We obtain an equivalent hydrogen column density of $0.74 \times 10^{22} \mathrm{~cm}^{-2}$; the same value was obtained by Christian \& Swank (1997) using the Einstein solidstate spectrometer (SSS; $0.5-4.5 \mathrm{keV}$ ), estimating a distance to the source of $5 \pm 1.5 \mathrm{kpc}$ (hereafter we will use this distance). Adopting this value as the distance to the source, the extrapolated unabsorbed luminosity in the $0.1-100 \mathrm{keV}$ energy range was $4.3 \times 10^{37} \mathrm{erg} \mathrm{s}^{-1}$.
Table 6. Best-fit values of the parameters obtained fitting the emission features with relativistic smeared lines (diskline in XSPEC). The Fe-K $\alpha$ feature is modelled with two diskline components.

\begin{tabular}{|c|c|c|c|}
\hline Parameters & Aver. spectrum & Spectrum $1+3$ & Spectrum 2 \\
\hline$N_{\mathrm{H}}\left(10^{22} \mathrm{~cm}^{-2}\right)$ & $0.734_{-0.004}^{+0.002}$ & 0.734 (fixed) & 0.734 (fixed) \\
\hline$k T_{\mathrm{BB}}(\mathrm{keV})$ & $1.801_{-0.009}^{+0.013}$ & $1.784_{-0.013}^{+0.025}$ & $1.820_{-0.011}^{+0.012}$ \\
\hline$N_{\text {BB }}\left(10^{-1}\right)$ & $1.043_{-0.011}^{+0.007}$ & $0.920_{-0.020}^{+0.013}$ & $1.123_{-0.010}^{+0.008}$ \\
\hline$k T_{\text {DIS квв }}(\mathrm{keV})$ & $1.06 \pm 0.02$ & $1.08_{-0.02}^{+0.03}$ & $1.056_{-0.017}^{+0.011}$ \\
\hline$N_{\text {DIS KBB }}$ & $201_{-14}^{+5}$ & $210_{-20}^{+14}$ & $186_{-5}^{+10}$ \\
\hline$E_{1 \mathrm{keV}}(\mathrm{keV})$ & $1.052_{-0.005}^{+0.006}$ & $1.032_{-0.017}^{+0.009}$ & $1.062_{-0.009}^{+0.007}$ \\
\hline$\sigma_{1 \mathrm{keV}}(\mathrm{eV})$ & $86_{-6}^{+13}$ & $120_{-8}^{+20}$ & $66_{-8}^{+10}$ \\
\hline$I_{1 \mathrm{keV}}$ & $24_{-2}^{+3}$ & $41_{-4}^{+9}$ & $17.0_{-1.4}^{+2.1}$ \\
\hline Equiv. width (eV) & $22 \pm 2$ & $31 \pm 3$ & $17 \pm 2$ \\
\hline$\beta$ emiss. index & $-2.2 \pm 0.2$ & $-2.17_{-0.19}^{+0.14}$ & $-2.10_{-0.19}^{+0.12}$ \\
\hline$R_{\text {in }}\left(R_{\mathrm{g}}\right)$ & $9_{-3}^{+17}$ & $6^{+14}$ & $9_{-3}^{+20}$ \\
\hline$R_{\text {out }}\left(R_{\mathrm{g}}\right)$ & $3600_{-1900}^{+18000}$ & 3600 (fixed) & 3600 (fixed) \\
\hline$\theta$ (degrees) & $43_{-3}^{+4}$ & 43 (fixed) & 43 (fixed) \\
\hline$E_{\mathrm{S} X \mathrm{XI}}(\mathrm{keV})$ & 2.62 (fixed) & 2.62 (fixed) & 2.62 (fixed) \\
\hline$I_{\mathrm{S} \mathrm{XVI}}$ & $2.0 \pm 0.5$ & $3.4_{-1.2}^{+1.0}$ & $0.7 \pm 0.6$ \\
\hline Equiv. width $(\mathrm{eV})$ & $5 \pm 3$ & $8 \pm 4$ & $2_{-2}^{+3}$ \\
\hline$E_{\text {Ar XVIII }}(\mathrm{keV})$ & $3.30_{-0.03}^{+0.04}$ & $3.32 \pm 0.05$ & $3.29 \pm 0.04$ \\
\hline$I_{\mathrm{Ar} X \mathrm{XVII}}$ & $3.2_{-0.8}^{+0.5}$ & $3.7_{-1.2}^{+0.9}$ & $2.6_{-0.7}^{+0.6}$ \\
\hline Equiv. width (eV) & $10_{-10}^{+4}$ & $12_{-5}^{+6}$ & $8_{-3}^{+5}$ \\
\hline$E_{\text {Ca XIX }}(\mathrm{keV})$ & $3.896 \pm 0.012$ & $3.89_{-0.04}^{+0.03}$ & $3.90 \pm 0.03$ \\
\hline$I_{\text {Ca XIX }}$ & $3.4_{-0.7}^{+0.5}$ & $3.6_{-1.1}^{+1.0}$ & $3.0 \pm 0.7$ \\
\hline Equiv. width (eV) & $14_{-8}^{+4}$ & $15_{-6}^{+5}$ & $12_{-6}^{+3}$ \\
\hline $\mathrm{E}_{\mathrm{Fe} \mathrm{XXV}}(\mathrm{keV})$ & 6.70 (fixed) & 6.70 (fixed) & 6.70 (fixed) \\
\hline$I_{\mathrm{Fe} \mathrm{XXV}}$ & $4.2_{-0.9}^{+0.4}$ & $4.9 \pm 1.3$ & $3.9_{-09}^{+1.0}$ \\
\hline Equiv. width (eV) & $40 \pm 10$ & $60_{-30}^{+20}$ & $40_{-20}^{+10}$ \\
\hline$E_{\mathrm{Fe} \text { XXVI }}(\mathrm{keV})$ & 6.97 (fixed) & 6.97 (fixed) & 6.97 (fixed) \\
\hline$I_{\mathrm{Fe} \text { XXVI }}$ & $1.6_{-0.3}^{+0.6}$ & $0.9_{-0.9}^{+0.9}$ & $2.1_{-0.7}^{+0.6}$ \\
\hline Equiv. width (eV) & $17_{-17}^{+9}$ & $10_{-10}^{+20}$ & $20 \pm 10$ \\
\hline$\chi_{\text {red }}^{2}$ (d.o.f.) & $1.49(268)$ & $1.25(271)$ & $1.30(271)$ \\
\hline
\end{tabular}

Note. See Table 5 .

\subsection{Spectral analysis of the selected time intervals}

To study possible changes of the broad emission line in the Fe$\mathrm{K} \alpha$ region of the XMM spectrum of GX 349+2, we extracted the EPIC-pn spectrum from interval 2 (Spectrum 2) and from intervals 1 and 3 together (Spectrum $1+3$ ). Since the $4-10 \mathrm{keV}$ lightcurve (Fig. 3, middle panel) indicates a higher count rate in time interval 2 than in intervals 1 and 3 , we expect a harder spectrum corresponding to interval 2 . The lower statistics in the time-selected spectra, due to the lower exposure times, requires that we fix some parameters of the diskline components. We fixed the outer radius and the inclination angle of the system to the values obtained fitting the spectrum corresponding to the whole observation. Initially we adopted only a diskline component to fit the $\mathrm{Fe}-\mathrm{K} \alpha$ broad feature, obtaining an energy value of $6.74 \pm 0.04$ and $6.78 \pm 0.03 \mathrm{keV}$ corresponding to Spectrum $1+3$ and 2, respectively. We note that while the line energy is compatible with Fe XXV transition for Spectrum 1+3, it is not compatible for Spectrum 2, which is when the count rate in the 
4-10 keV energy band increases. The best-fit values are reported in Table 5 (Cols. 3 and 4).

As a consequence of this result, we fitted the Fe-K $\alpha$ broad feature in Spectra 2 and 1+3 adopting two diskline components with energies fixed at 6.7 and $6.97 \mathrm{keV}$. Also in this case we fixed the outer radius and the inclination angle of the system to the values obtained fitting the average spectrum. The best-fit values are reported in Table 6 (Cols. 3 and 4). We find that the main differences between Spectrum 1+3 and Spectrum 2 is the value of the blackbody normalization that is larger in Spectrum 2. Also we note that the flux of the line at $1 \mathrm{keV}$, the S XVI line, the Ar XVIII line, the CaXIX line, and Fe XXV line is lower in Spectrum 2 than Spectrum 1+3 while the flux of the Fe XXVI line is larger in Spectrum 2 than Spectrum 1+3. In the following section we discuss these results.

\section{Discussion}

We performed a spectral analysis of a $22.5 \mathrm{ks}$ XMM observation of GX $349+2$ in the $0.7-10 \mathrm{keV}$ energy range. The large flux from the source caused a telemetry overflow in the EPIC-pn data collection, resulting in an effective exposure time of $10 \mathrm{ks}$. We fit the continuum emission, adopting a blackbody plus a multicolored disk blackbody and both these components are absorbed by neutral matter. Five emission features were clearly visible in the spectrum.

Initially we fitted these features using Gaussian profiles and finding their energies at 1.05, 2.62 (fixed), 3.32, 3.9, and $6.8 \mathrm{keV}$. The corresponding widths and equivalent width were 90, 140 (fixed), 190, 100, and $280 \mathrm{eV}$, respectively, and 22, 6, 12,9 and $49 \mathrm{eV}$, respectively. We associated the first four lines with L-shell Fe XXII-XXIII transition, Ly- $\alpha$ S XVI, Ly- $\alpha$ Ar XVIII, and Ca XIX resonance transition, respectively. The broader emission feature in the $\mathrm{Fe}-\mathrm{K} \alpha$ region was not identifiable, hence we supposed that it is a blending of Fe XXV and Fe XXVI emission lines. Fitting the broad feature adopting two Gaussian profiles centered at 6.7 and $6.97 \mathrm{keV}$ we obtained that their corresponding widths were 220 and $280 \mathrm{eV}$. We conclude that if the Fe-k $\alpha$ feature is a blend of Fe XXV and Fe XXVI emission lines, these lines are intrinsically broad.

BeppoSAX observed three emission lines in the spectrum of GX 349+2 (Iaria et al. 2004; Di Salvo et al. 2001): the first one centered at $1.18 \pm 0.03 \mathrm{keV}$, interpreted as the L-shell Fe XXIV transition, the second at $2.60 \pm 0.06 \mathrm{keV}$, interpreted as the Ly$\alpha \mathrm{S}$ XVI transition and, finally, the third at $6.75 \pm 0.01 \mathrm{keV}$, interpreted as the Fe XXV transition. In this observation, we find that the centroid of the broad emission line at $1 \mathrm{keV}$ is at a lower energy, suggesting an L-shell transition of less ionized iron (Fe XXII-XXIII) although we cannot exclude a more complex blending of Fe XXI-XXIV. The widths and equivalent widths of these three lines are very similar comparing our observation and the two BeppoSAX observations of GX 349+2 (Iaria et al. 2004; Di Salvo et al. 2001) in the non-flaring state. Iaria et al. (2004) found, during interval 2 in the non-flaring state, widths of $<80,<120$ and $210 \pm 80 \mathrm{eV}$, and equivalent widths of $23 \pm 8$, $7_{-1}^{+4}$, and $52 \pm 11 \mathrm{eV}$ corresponding to the Fe XXIV, S XVI, and Fe XXV emission lines. Furthermore, Di Salvo et al. (2001) obtained equivalent widths for the Fe-K $\alpha$ line of $71 \mathrm{eV}$ and $34 \mathrm{eV}$, in the non-flaring and flaring state of the source, respectively. From these rough comparisons it seems that the source is in a non-flaring state during the observation discussed in this work, although we cannot assert this conclusion without an accurate analysis of the time variabilities.
These emission features can be fitted slightly better adopting smeared relativistic lines. The large widths of the lines suggest that these are produced by reflection of the primary spectral component by the accretion disk. Similar broad emission features, discussed as smeared lines, were observed in several systems containing a neutron star, such as Ser X-1 (Bhattacharyya \& Strohmayer 2007), SAX J1808.4-3658 (Papitto et al. 2009; Cackett et al. 2009a), 4U 1705-44 (Di Salvo et al. 2005, 2009), GX 340+0 (D'Aì et al. 2009), 4U 1636-536 (Pandel et al. 2008), X 1624-490 (Iaria et al. 2007), and 4U 1820-30 and GX 349+2 (Cackett et al. 2008), and the authors of these papers agree in interpreting the broadening as due to relativistic and Doppler effects. In the following, we show the self-consistency of the disk reflection scenario, discussing the parameters reported in Table 6.

We infer the inner radius of the accretion disk, $R_{\text {disk }}$, using the normalization value of the multicolored disk blackbody and the inclination angle of the source, $i=43_{-3}^{+4} \mathrm{deg}$, obtained by the relativistic smeared line, finding that $R_{\text {disk }}$ is $8 \pm 3$ and $8 \pm 2$ $\mathrm{km}$ for Spectrum $1+3$ and 2, respectively. The blackbody radius, $R_{\mathrm{BB}}$, inferred by the best-fit values of the blackbody normalization, is $4.2 \pm 1.4$ and $4.5 \pm 1.4 \mathrm{~km}$ for Spectrum $1+3$ and 2 , respectively, too small to be associated with the X-ray emission from the NS surface.

Taking into account possible modifications of the multicolored disk blackbody component due to electron scattering (Shakura \& Syunyaev 1973; White et al. 1988), the measured color temperature, $T_{\mathrm{col}}$, is related to the effective temperature of the inner disk, $T_{\text {col }}=f T_{\text {eff }}$, where $f$ is the spectral hardening factor, and $R_{\text {eff }}=f^{2} R_{\text {measured }}$. Adopting $f=1.7$ as estimated by Shimura \& Takahara (1995) for a luminosity around $10 \%$ of the Eddington limit, close to the $25 \%$ of the Eddington limit estimated for GX 349+2 in our analysis, the corrected value of $R_{\text {disk }}$ is $24 \pm 7 \mathrm{~km}$. The radius of the accretion disk is compatible with the value of the inner radius obtained by the relativistic smeared lines, that is, assuming a neutron star mass of $1.4 M_{\odot},<41 \mathrm{~km}$ and $19_{-6}^{+41} \mathrm{~km}$, for Spectrum $1+3$ and 2 , respectively. In the same way, correcting the radius of the blackbody component for the electron scattering, we infer that $R_{\mathrm{BB}}=13 \pm 4 \mathrm{~km}$. This radius is compatible with the NS radius and suggests that the blackbody component is produced very close the NS surface. In this scenario, we directly observe the emission from inside the inner radius of the accretion disk, that is close the NS surface, and the blackbody component is interpreted as the direct emission from a compact corona and/or a boundary layer (BL) located between the neutron star and the inner radius of the disk. We should expect a Comptonized emission from the compact corona and/or $\mathrm{BL}$, but if it is optically thick the Comptonized component results saturated and mimics a blackbody emission in the adopted energy range.

We observe, for the first time, reflection from the inner accretion disk that involves not only the K-transitions of iron but also ions of lighter elements such as sulfur, argon and calcium which all correspond to a similar ionization parameter. We observe emission lines associated with S XVI, Ar XVIII, Ca XIX, and Fe XXV, which correspond to an ionization parameter $\log (\xi) \simeq$ 3. A similar detection was reported by Di Salvo et al. (2009) analysing XMM data of 4U 1705-44.

Using the relation $\xi=L_{\mathrm{x}} /\left(n_{\mathrm{e}} r^{2}\right)$, where $L_{\mathrm{x}}$ is the unabsorbed luminosity in the $0.1-100 \mathrm{keV}, n_{\mathrm{e}}$ the electron density of the plasma, and $r$ the distance from the source of the emitting plasma we can roughly estimate the electron density $n_{\mathrm{e}}$ adopting as distance from the source the inner and outer accretion disk radius, $R_{\text {in }}$ and $R_{\text {out }}$, obtained from the diskline components. We obtain 
that the electron density $n_{\mathrm{e}}$ decreases from $10^{22}$ to $10^{17} \mathrm{~cm}^{-3} \mathrm{go}-$ ing from the inner to the outer radius. These values of electron densities are in agreement with the values reported by Vrtilek et al. (1993), who estimated the electron density of an extended cloud to be $10^{22} \mathrm{~cm}^{-3}$ near the equatorial plane at a distance from the neutron star surface of less than $10^{8} \mathrm{~cm}$ (see Fig. 2 in Vrtilek et al. 1993).

From the analysis of the two selected spectra, we find that the extrapolated (not absorbed) flux in the $0.1-100 \mathrm{keV}$ band of the multicolored disk blackbody is approximately constant: $(0.61 \pm 0.12) \times 10^{-8}$ and $(0.49 \pm 0.05) \times 10^{-8} \mathrm{erg} \mathrm{cm}^{-2} \mathrm{~s}^{-1}$ for Spectrum $1+3$ and 2, respectively. The blackbody component has a larger flux in Spectrum $2\left(0.94 \pm 0.03 \times 10^{-8} \mathrm{erg} \mathrm{cm}^{-2} \mathrm{~s}^{-1}\right)$ than in Spectrum $1+3\left(0.77 \pm 0.02 \times 10^{-8} \mathrm{erg} \mathrm{cm}^{-2} \mathrm{~s}^{-1}\right)$. This suggests that the intensity change during the observation is driven by the blackbody component. In our scenario the blackbody component is associated with the emission from a BL around the compact object and this emission illuminates the surface of the inner accretion disk producing the reflection lines observed in the spectra. We note that the line fluxes are higher in Spectrum 1+3 than in Spectrum 2 except for the flux associated with the Fe XXVI line which shows an anticorrelated behaviour.

Our scenario may easily explain these results: in fact, if the emission from the BL increases, the flux illuminating the accretion disk increases, then the ionization parameter of the reflecting matter also increases under the hypothesis that the density of the matter does not change. This implies that transitions of heavier ions are more probable, since lighter ions such as S XVI, Ar XVIII, Ca XIX, and Fe XXV decrease in number while heavier ions such as Fe XXVI increase in number, giving a larger flux of the Fe XXVI line and a lower flux of the other lines.

GX $349+2$ is a NS LMXBs belonging to the Z-class sources and its behaviour is very similar to the prototype of its class, Sco X-1. Steeghs \& Casares (2002) analysed the optical spectrum of Sco X-1 and obtained a firm mass ratio limit of $q \lesssim 0.61$ from the phase-resolved spectroscopy, where $q$ is the ratio of the companion star mass to the neutron star mass. If we assume that the optical periodicity of $21.9 \pm 0.4 \mathrm{~h}$, observed in GX 349+2 by Wachter \& Margon (1996), is the orbital period of the system, the mass function of GX 349+2 is $f(M)=0.032 M_{\odot}$ (see Wachter \& Margon 1996). We find that the inclination angle of the system is $43_{-3}^{+4} \mathrm{deg}$. Assuming a neutron star mass of $1.4 M_{\odot}$ we find $q \sim 0.56$, a value very similar to the upper limit estimated for Sco X-1, giving a mass of the companion star of $M_{\mathrm{c}}=0.78_{-0.06}^{+0.07} M_{\odot}$, typical for a NS LMXB of the Z class $(q \leq 0.61$ for Sco X-1, Steeghs \& Casares 2002; $q=0.34 \pm 0.04$ for Cyg X-2, Casares et al. 1997).

Furthermore Sco X-1 shows twin compact radio lobes forming an angle, with respect the line of sight, of $44 \pm 6$ deg (error at $1 \sigma$, see Fomalont et al. 2001). If the jet from the source producing the radio lobes is almost perpendicular to the equatorial plane of the system then the angle of $44 \pm 6$ deg corresponds to the inclination angle of the system, similar to the inclination angle of $43_{-3}^{+4}$ deg obtained for GX $349+2$ in this work. Our results indicate that Sco X-1 and GX 349+2 are very similar sources, as already suggested by other authors (see e.g. Kuulkers \& van der Klis 1998).

Although our scenario looks reasonable, our values of the diskline parameters are not compatible with the recent ones reported by Cackett et al. (2008). Cackett et al., analysing Suzaku data of GX 349+2, fitted the broad emission feature in the Fe$\mathrm{K} \alpha$ region adopting a smeared relativistic line with the outer radius fixed at $1000 R_{\mathrm{g}}$. They found a line energy associated with Fe XXVI, an inner radius of $16.5 \pm 0.8 \mathrm{~km}$, an emissivity index of $-4.1 \pm 0.3$ and an inclination angle of the system of $23^{\circ} \pm 1^{\circ}$. The differences in line energy, emissivity index, and inner radius of the reflecting skin could be explained supposing that GX $349+2$ was observed in a different spectral state. We find that the $\mathrm{Fe}-\mathrm{K} \alpha$ broad emission feature is associated with the Fe XXV line or a blending of Fe XXV and Fe XXVI in which the Fe XXV line is more intense. Our measurement is similar to the previous BeppoSAX observations of GX 349+2 (Di Salvo et al. 2001; Iaria et al. 2004) and to the two recent Chandra observations of this source (Cackett et al. 2009b). In all these observations GX $349+2$ was in NB/FB and/or in the bottom part of the FB. The only exception is the Suzaku observation of GX 349+2 (Cackett et al. 2008) where the broad emission line seems associated with Fe XXVI, suggesting that the source was in a different state; in fact, as discussed by Cackett et al., it is possible that GX 349+2 was in NB during that observation.

However, the different inclination angles of the system obtained in this work and by Cackett et al. (2008) cannot be easily explained since the inclination angle of the system should not depend on the state of the source and should not change. Using the inclination angle obtained by Cackett et al. we obtain a mass ratio $q \sim 1.25$ and a companion star mass of $1.74_{-0.11}^{+0.12} M_{\odot}$ for a neutron star mass of $1.4 M_{\odot}$, that is quite unusual for NS LMXBs.

Higher statistics spectroscopic studies are needed to put tighter constraints on the system parameters of this source, since these can give important information on the whole binary system and on the physics of the accretion flow close to the compact object.

\section{Conclusions}

We analysed a XMM observation of GX 349+2, finding the presence of prominent emission features associated with S XVI, Ar XVIII, Ca XIX, Fe XXV and, possibly, Fe XXVI. The emission features can be fitted with relativistic smeared lines (diskline). The continuum emission was fitted adopting a multicolored disk blackbody plus a blackbody component. We investigated the scenario in which the broad emission lines are formed by reflection from an ionized skin in the inner region of the accretion disk illuminated by the emission of a compact corona (or a boundary layer) surrounding the neutron star that we fitted with a blackbody component with a temperature of $1.8 \mathrm{keV}$. We find a selfconsistent explanation of our results. The inner radius of the disk blackbody is $24 \pm 7 \mathrm{~km}$ while the broad lines give an inner disk radius of less than $40 \mathrm{~km}$ from the neutron star center. We discuss that the electron density of the reflecting plasma is between $10^{17}$ and $10^{22} \mathrm{~cm}^{-3}$ and that the inclination angle of the source is around $43^{\circ}$. Finally, we infer that, for an inclination angle of $43^{\circ}$, the mass of the companion star is $0.78 M_{\odot}$.

Acknowledgements. We are very grateful to the referee for his/her suggestions and comments.

\section{References}

Bhattacharyya, S., \& Strohmayer, T. E. 2007, ApJ, 664, L103

Cackett, E. M., Miller, J. M., Bhattacharyya, S., et al. 2008, ApJ, 674, 415

Cackett, E. M., Altamirano, D., Patruno, A., et al. 2009a, ApJ, 694, L21

Cackett, E. M., Miller, J. M., Homan, J., et al. 2009b, ApJ, 690, 1847

Casares, J., Charles, P., \& Kuuklers, E. 1997, NASA STI/Recon Technical Report N, 98, 99651

Christian, D. J., \& Swank, J. H. 1997, ApJS, 109, 177

D’Aì, A., Iaria, R., Di Salvo, T., Matt, G., \& Robba, N. R. 2009, ApJ, 693, L1

den Herder, J. W., Brinkman, A. C., Kahn, S. M., et al. 2001, A\&A, 365, L7

Di Salvo, T., D'Ai', A., Iaria, R., et al. 2009, ArXiv e-prints 
Di Salvo, T. Iaria, R., Méndez, M., et al. 2005, ApJ, 623, L121

Di Salvo, T., Robba, N. R., Iaria, R., et al. 2001, ApJ, 554, 49

Fomalont, E. B., Geldzahler, B. J., \& Bradshaw, C. F. 2001, ApJ, 558, 283

Hasinger, G., \& van der Klis, M. 1989, A\&A, 225, 79

Hasinger, G., van der Klis, M., Ebisawa, K., Dotani, T., \& Mitsuda, K. 1990, A\&A, 235, 131

Iaria, R., Di Salvo, T., Robba, N. R., et al. 2004, ApJ, 600, 358

Iaria, R., Lavagetto, G., D’Aí, A., di Salvo, T., \& Robba, N. R. 2007, A\&A, 463 289

Jansen, F., Lumb, D., Altieri, B., et al. 2001, A\&A, 365, L1

Kuulkers, E., \& van der Klis, M. 1998, A\&A, 332, 845

O'Neill, P. M., Kuulkers, E., Sood, R. K., \& Dotani, T. 2001, A\&A, 370, 479

O’Neill, P. M., Kuulkers, E., Sood, R. K., \& van der Klis, M. 2002, MNRAS, 336,217
Pandel, D., Kaaret, P., \& Corbel, S. 2008, ApJ, 688, 1288

Papitto, A., di Salvo, T., D’A1, A., et al. 2009, A\&A, 493, L39

Shakura, N. I., \& Syunyaev, R. A. 1973, A\&A, 24, 337

Shimura, T., \& Takahara, F. 1995, ApJ, 445, 780

Steeghs, D., \& Casares, J. 2002, ApJ, 568, 273

Strüder, L., Briel, U., Dennerl, K., et al. 2001, A\&A, 365, L18

Turner, M. J. L., Abbey, A., Arnaud, M., et al. 2001, A\&A, 365, L27

van der Klis, M. 1995, in Flares and Flashes, Lecture Notes in Physics (Berlin:

Springer Verlag), ed. J. Greiner, H. W. Duerbeck, \& R. E. Gershberg Lecture Notes in Physics, 454, IAU Colloq., 151, 321

Vrtilek, S. D., Soker, N., \& Raymond, J. C. 1993, ApJ, 404, 696

Wachter, S., \& Margon, B. 1996, AJ, 112, 2684

White, N. E., Stella, L., \& Parmar, A. N. 1988, ApJ, 324, 363

Zhang, W., Strohmayer, T. E., \& Swank, J. H. 1998, ApJ, 500, L167 